\title{
THz SIS mixers with normal-metal AI tuning circuits
}

\author{
M Bin $\dagger$, M C Gaidis $\dagger$, J Zmuidzinas $\dagger$, T G Phillips $\dagger$ and \\ H G Leduc $\ddagger$
}

† G W Downs Laboratory of Physics, 320-47, California Institute of Technology, Pasadena, CA 91125, USA

$\ddagger$ Jet Propulsion Laboratory, 302-231, Pasadena, CA 91101, USA

Received 18 October 1995, in final form 1 December 1995

\begin{abstract}
Nb-based superconductor-insulator-superconductor (SIS) mixers with $\mathrm{Nb}$ tuning circuits have demonstrated good results up to the $\mathrm{Nb}$ gap frequency. Above the gap frequency the performance is expected to degrade quickly because $\mathrm{RF}$ loss in $\mathrm{Nb}$ becomes significant. In this paper we present the results of an effort to extend $\mathrm{Nb}$-based SIS mixers to $\mathrm{THz}$ frequencies by employing lower-loss normal-metal Al wiring and tuning structures. The SIS mixer has two $\mathrm{Nb} / \mathrm{Al}$-oxide/Nb junctions connected by an Al microstrip inductor. The direct detection response of the device was measured by a Fourier transform spectrometer. A double-side-band receiver noise temperature of $840 \mathrm{~K}$ was obtained at $1042 \mathrm{GHz}$ when the device was operated at $2.5 \mathrm{~K}$.
\end{abstract}

\section{Introduction}

Quasi-optical SIS Nb/Al-oxide/Nb mixers with integrated $\mathrm{Nb}$ tuning circuits have achieved good performance up to the $\mathrm{Nb}$ gap frequency $(\approx 700 \mathrm{GHz})$ and a little beyond [1,2]. The tuning circuits, made from microstrip transmission lines, serve to tune out the junction capacitance at the operating frequency and to match the impedance of the junction to that of the integrated antenna. Below the gap frequency $\mathrm{Nb}$ has very small RF losses as a superconductor. Above the gap frequency, RF losses increase significantly due to pair breaking in $\mathrm{Nb}$. Since $\mathrm{Nb}$ is a poor normal conductor, we expect that replacing $\mathrm{Nb}$ with a good normal conductor (e.g. $\mathrm{Au}, \mathrm{Ag}$ or $\mathrm{Al}$ ) as the wiring element can result in a better mixer performance above the gap frequency [9].

To better understand the role of microstrip losses, we have simulated the direct detection response of our mixer design and compared devices with $\mathrm{Nb}$ wiring and $\mathrm{Al}$ wiring. If a residual resistivity ratio of $10\left(\rho_{300 \mathrm{~K}} / \rho_{4} \mathrm{~K}\right)$ is assumed for $\mathrm{Al}$, the two mixers offer comparable response around $900 \mathrm{GHz}$ but the Al-wired device becomes superior at higher frequencies.

\section{Simulation}

Our approach to mixer design is to optimize the RF coupling into the junction resistance within the desired bandwidth. Using Fourier transform spectroscopy (FTS), design ideas can be easily checked since the direct detector response from the FTS yields the RF coupling efficiency. Below we present the simulations of the expected RF

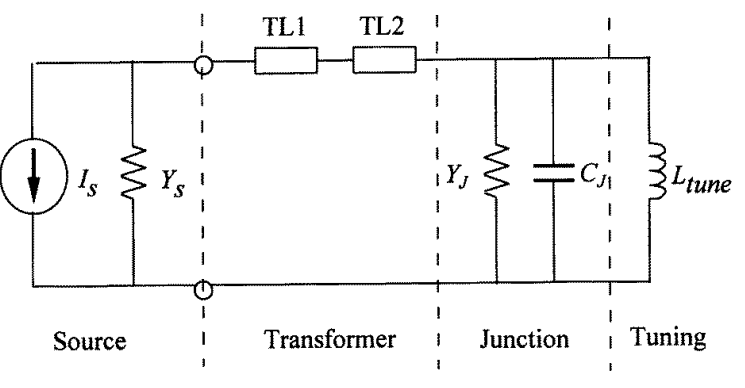

Figure 1. The equivalent circuit for a twin-slot antenna double-junction mixer design. The antenna is represented by the current source at the left-hand side. The junction and tuning elements are represented by the right-hand side of the circuit.

coupling into our double-junction mixers on twin-slot antennas with antisymmetric feeds [2].

\subsection{Mixer equivalent circuit}

The relevant portion of the equivalent circuit of our mixers is shown in figure 1 [2]. The antenna behaves like a current source with source admittance $Y_{\mathrm{S}}$ (calculated by the method of moments [3]). $L_{\text {tune }}$ is the microstrip inductor serving to tune out the junction capacitance $C_{J} . Y_{J}$ is the junction's RF admittance and is calculated from Tucker's theory based on the junction's $I-V$ curve [4]. There are either one or two sections of microstrip transmission lines TL1 and TL2 used as transformers to match the impedance of the junction to that of the antenna. 


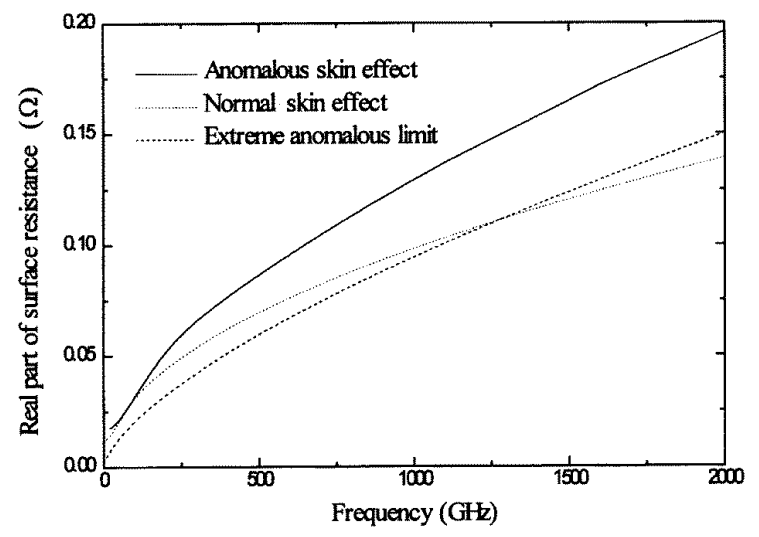

Figure 2. The surface impedance as a function of frequency calculated for an Al thin film of thickness $0.2 \mu \mathrm{m}$. The resistivity is $2.45 \mu \Omega \mathrm{cm}$ and the mean free path is $0.016 \mu \mathrm{m}$ at room temperature. The residual resistance ratio is assumed to be 10 from room temperature to $4.2 \mathrm{~K}$.

\subsection{A two-port network simulator}

To be able to accurately simulate the lossy microstrip line in our devices, we have developed a computer program to analyse general microwave circuits in a two-port fashion. Using this program we can optimize our device design to maximize RF coupling into the junction. The RF coupling $\eta_{\mathrm{RF}}$ is defined as the ratio of absorbed power at the junction to the available power at the source. In the special case when the transmission lines are lossless

$$
\eta_{\mathrm{RF}}=1-\frac{\left|Y_{R}-Y_{S}^{*}\right|^{2}}{\left|Y_{R}+Y_{S}\right|^{2}}
$$

where $Y_{R}$ is the admittance presented by the portion of the circuit in figure 1 to the right of the source. Practical transmission lines are not lossless and $\eta_{\mathrm{RF}}$ will be reduced. To accurately include the transmission lines in our simulation we follow the procedure of [3]. A first calculation is made by assuming perfect conductors. Then we replace the perfect conductor with metals of finite conductivity by introducing an extra contribution $g Z_{S}$ to the series impedance of the line. Here $g$ is a geometrical factor and $Z_{S}$ denotes the surface impedance of the metal.

For normal conductors at cryogenic temperatures and $\mathrm{THz}$ frequencies, the electron mean free path $l$ is often long compared with the penetration depth $\delta$ and comparable with the thickness $d$ of the transmission line films. Under such conditions, the theory of the anomalous skin effect should be used in calculating the surface impedance. We calculate $Z_{S}$ for $\mathrm{Al}$ films by solving the integrodifferential equation (2.7) in [5]. The results obtained for the anomalous case, the extreme anomalous case $(d \rightarrow \infty$ and $l \gg \delta$ ) and the normal skin effect case are compared in figure 2.

For superconductors the surface impedance should be calculated by solving the Mattis-Bardeen theory [6] for a thin film as done by Pöpel [7]. This process can be simplified somewhat if the films are thick enough to use a bulk approximation. In our calculations we further

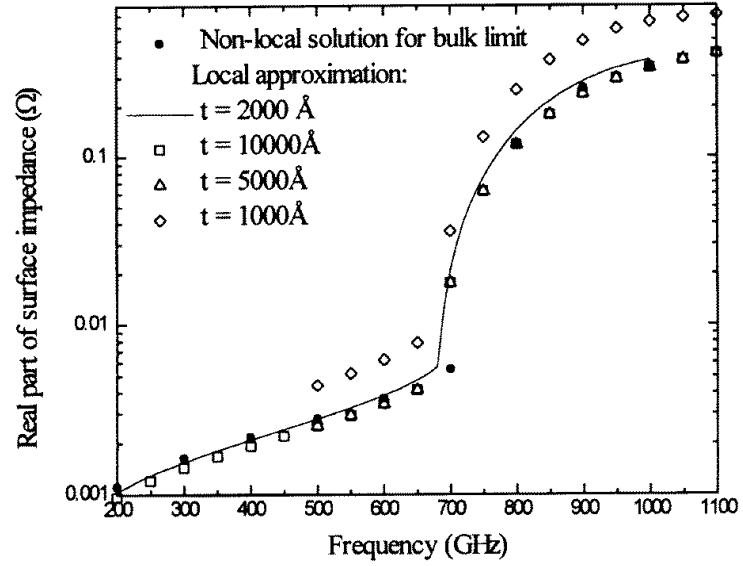

Figure 3. Surface impedance calculated for superconducting $\mathrm{Nb}$ film. Comparison is made between exact solutions of the Mattis-Bardeen theory in the bulk limit and the local approximations for different film thicknesses.

simplify by using the Mattis-Bardeen theory in the extreme anomalous limit. Results thus obtained are compared with exact solutions of the Mattis-Bardeen theory in the bulk limit [8] in figure 3 . The $4.2 \mathrm{~K} \mathrm{Nb}$ electron mean free path is $\sim 11 \mathrm{~nm}$ and penetration depth is $\sim 86 \mathrm{~nm}$. The results from our approximation for a $200 \mathrm{~nm}$ film are very close to those given by [8].

\subsection{Simulation results}

Using the simulation described above, we have designed and fabricated $\mathrm{Nb}$-wired SIS mixers optimized at $750 \mathrm{GHz}$. The simulated RF coupling and FTS measurements are presented in figure 4 . The parameters used in the simulation are given in table 1 . The junction normal state resistance was determined upon cool down to be approximately $20 \%$ larger than the design value but this has little effect on the frequency response. The junction capacitance was inferred by comparing the FTS data to the simulated response. The simulation predicts good RF coupling up to $800 \mathrm{GHz}$. Above $800 \mathrm{GHz}$ the loss is somewhat higher than predicted. In part this is caused by antenna loss which has not been included in the present simulation. Figure 5 shows a simulation and FTS measurement for an Al-wired mixer. This photomask design was originally made for an $\mathrm{Nb}$ wired circuit, but experiments with Al fabrication were carried out on this photomask set, then tested with the FTS to gauge our understanding of the performance of the microstrip line. The only assumption here is the residual resistance ratio of $\mathrm{Al}$, for which we used 10 , and the junction specific capacitance, $85 \mathrm{fF} \mu \mathrm{m}^{-2}$. The fit is reasonable, but again deviates at high frequencies. This is probably the result of errors in the parameter values used in the simulation because the surface resistance of normal metals should not vary rapidly with frequency.

Based on the previous experiments we have designed Al-wired SIS mixers for work at $1050 \mathrm{GHz}$. The RF coupling of one of the designs is shown in figure 6, along with a simulation for an $\mathrm{Nb}$-wired mixer optimized for best performance at $1050 \mathrm{GHz}$. 
Table 1. Parameters used in the simulations.

\begin{tabular}{ll}
\hline Junction capacitance of Nb-wired mixer & $C=146 \mathrm{fF}$ \\
Junction area of Nb-wired mixer & $A=2.25 \mu \mathrm{m}^{2}$ \\
Junction capacitance of Al-wired mixer & $C=191 \mathrm{fF}$ \\
Junction area of Al-wired mixer & $A=2.25 \mu \mathrm{m}^{2}$ \\
Junction normal resistance & $R_{N}=11 \Omega$ \\
Al resistivity at room temperature & $\rho_{N}=2.45 \mu \Omega \mathrm{cm}$ \\
Al mean free path at room-temperature & $I=16 \mathrm{~nm}$ \\
Superconducting Nb film thickness & $d=200 \mathrm{~nm}$ \\
Normal state resistance just above $T_{\mathrm{C}}$ & $\rho_{N}=5 \mu \Omega \mathrm{cm}$ \\
Critical temperature of Nb & $T_{C}=9.2 \mathrm{~K}$ \\
Gap voltage of Nb at $\mathrm{K}$ & $V_{g a p}=2.9 \mathrm{mV}$ \\
Dielectric constant of insulation layer SiO & $\varepsilon=5.6 \varepsilon_{0}$
\end{tabular}

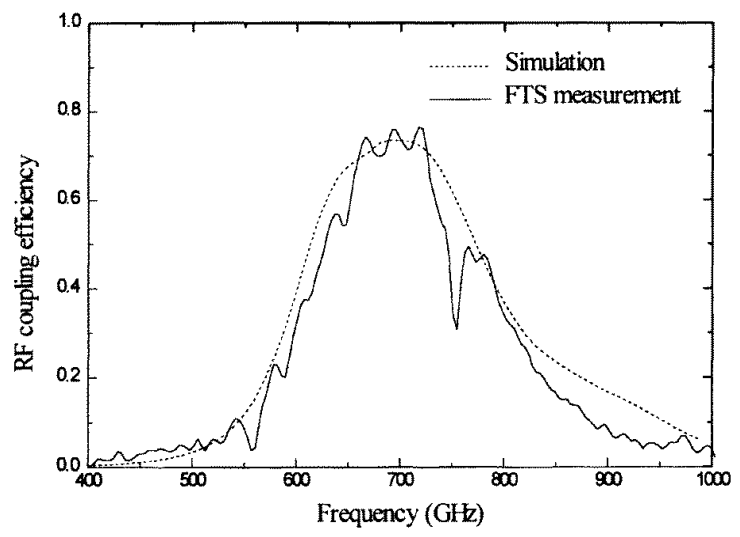

Figure 4. FTS measurements and simulations of direct response on an $\mathrm{Nb}$-wired mixer. Parameters used for the simulations are given in table 1.

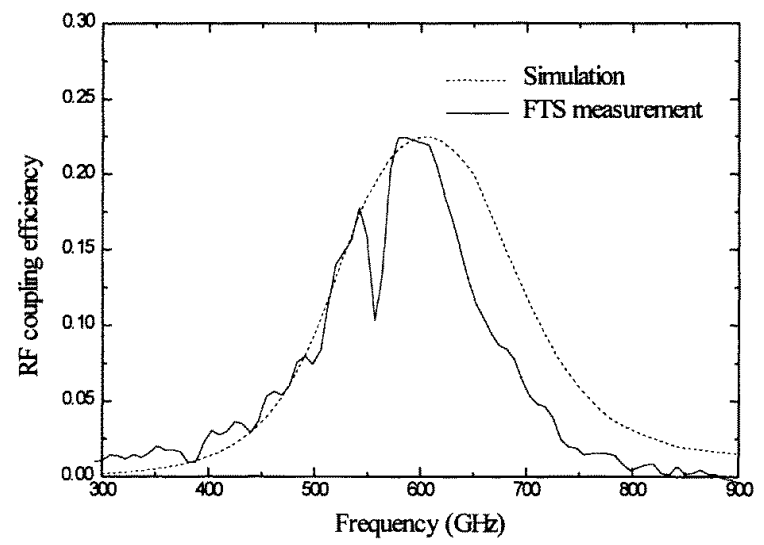

Figure 5. FTS measurements and simulations of direct response on an Al-wired mixer. Parameters used for the simulations are given in table 1.

\section{Experiments}

We have fabricated Al-wired Nb SIS mixers. We started from the four layers $\mathrm{Al} / \mathrm{Nb} / \mathrm{Al}$-oxide/ $\mathrm{Nb}$. The bottom $\mathrm{Al}$ layer was patterned into the antenna. The junctions were made by etching through the top trilayers. A $2000 \AA$ $\mathrm{SiO}$ insulating film was deposited for the microstrip tuning inductor and $4000 \AA \mathrm{SiO}$ for the impedance transformer. $\mathrm{Al}$

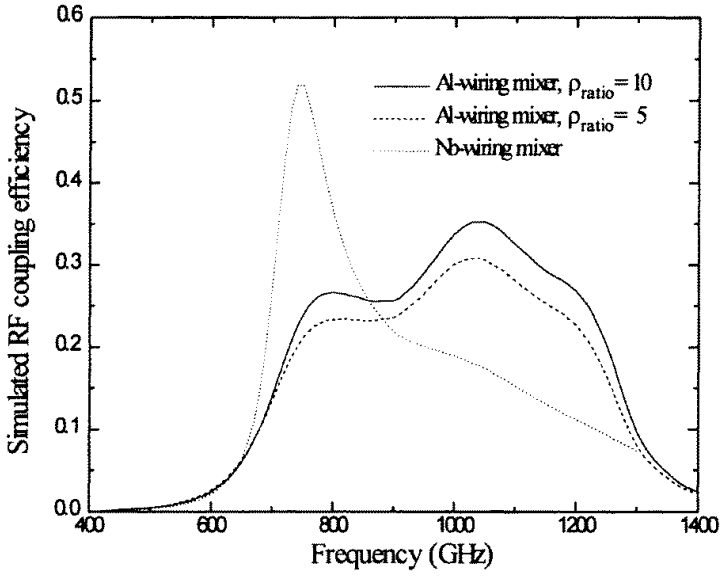

Figure 6. Simulations of direct detection response for Alor $\mathrm{Nb}$-wired mixers optimized at a working frequency of $1050 \mathrm{GHz}$. Residual resistivity ratios of 10 and 5 are assumed here.

wiring $2000 \AA$ thick was deposited as the last step. Typical d.c. $I-V$ curves have a sloped supercurrent, revealing a resistance in series with the junctions. This resistance is caused by the resistive current path through the $\mathrm{Al}$ microstrip and coplanar lines. We derived a residual resistivity ratio of 4.8 from the stripline geometry and the series resistance.

Standard $Y$-factor method was used to obtain our receiver noise temperature. The receiver consists of a $10 \mu \mathrm{m}$ Mylar beamsplitter for LO, a $25 \mu \mathrm{m}$ thick Mylar window, a $2.2 \mathrm{~mm}$ thick quartz IR filter at $77 \mathrm{~K}$, a polyethylene lens at $4 \mathrm{~K}$ and an antireflection-coated silicon hyperhemispherical lens. The mixer chip was glued to the hyperhemispherical lens. The intermediate frequency (IF) power was measured in a $1 \mathrm{GHz}$ bandwidth centred at 1.5 GHz. A Far-IR $\mathrm{CH}_{2} \mathrm{~F}_{2}$ gas laser pumped by a $\mathrm{CO}_{2}$ laser provided an LO source at $1042 \mathrm{GHz}$. The initial test with a device optimized at $1050 \mathrm{GHz}$ gave an uncorrected doublesideband receiver noise of $840 \mathrm{~K}$ at a bath temperature of $2.5 \mathrm{~K}$.

\section{Conclusion}

We have designed and fabricated Al-wired and Nb-based SIS mixers. The anomalous skin effect was considered for calculating the normal-metal $\mathrm{Al}$ transmission lines. 
The mixer design was optimized by maximizing the RF coupling efficiency at the frequency bandwidth of interest. Comparisons between simulated RF coupling responses of $\mathrm{Nb}$-wired and Al-wired mixers were given. Initial heterodyne tests of our $\mathrm{Al}$-wired mixers resulted in an uncorrected double-sideband receiver noise temperature of $840 \mathrm{~K}$ at $1042 \mathrm{GHz}$ when the device was working at $2.5 \mathrm{~K}$. Our work demonstrates that $\mathrm{Nb}$ junctions can offer superior performance at terahertz frequencies when implemented with low-loss normal-metal Al tuning structures.

\section{Acknowledgments}

We thank Paul Stockman and Geoff Blake for their assistance with the far-IR laser measurements. This work was supported by NASA grants NAG2-744 and NAGW107, the NASA/JPL Center for Space Microelectronics Technology, and an NSF Presidential Young Investigator grant to J Zmuidzinas.

\section{References}

[1] Zmuidzinas J, LeDuc H G, Stern J A and Cypher S R 1994 IEEE Trans. Microwave Theory Tech. 42 698-706

[2] Gaidis M C, Bin M, Miller D, Zmuidzinas J, Leduc H G and Stern J A 1995 Proc. 6th Int. Symp. on Space THz Technology (21-23 March 1995, Caltech, Pasadena, CA) ed J Zmuidzinas (Pasadena: California Institute of Technology) pp 305-13

[3] Zmuidzinas J and LeDuc H G 1992 IEEE Trans. Microwave Theory Tech. 40 1797-804

[4] Tucker T R and Feldman M J 1985 Rev. Mod. Phys. 57 1055-113

[5] Kautz R L 1979 J. Res. NBS 84 247-59

[6] Mattis D C and Bardeen J 1958 Phys. Rev. 11 412-17

[7] Pöpel R 1989 Superconducting Quantum Electronics (Berlin: Springer) p 44

[8] Pöpel $\mathrm{R}$ private communication

[9] Van de Stadt H et al 1995 Proc. 6th Int. Symp. on Space THz Technology (21-23 March 1995, Caltech, Pasadena, $C A$ ) ed J Zmuidzinas (Pasadena: California Institute of Technology) pp 66-7 